Tropical Journal of Pharmaceutical Research March 2021; 20 (3): 519-524

ISSN: $1596-5996$ (print); 1596-9827 (electronic)

(C) Pharmacotherapy Group, Faculty of Pharmacy, University of Benin, Benin City, 300001 Nigeria.

Available online at http://www.tjpr.org

Original Research Article

http://dx.doi.org/10.4314/tjpr.v20i3.11

\title{
Nuclear protein 1 promotes unfolded protein response during endoplasmic reticulum stress, and alleviates apoptosis induced by cisplatin in non-small cell lung cancer cells
}

\author{
Yinhui Sun ${ }^{1}$, Lihuai Wang ${ }^{2 *}$, Changqing Deng ${ }^{3}$, Hua Liu², Zhongcong Guo², \\ Zelin Chang $^{3}$, Jianxiong Mo $^{3}$ \\ ${ }^{1}$ Medical College, Hunan University of Traditional Chinese Medicine, Changsha City, ${ }^{2}$ Department of Oncology, The First \\ Affiliated Hospital of Hunan University of Traditional Chinese Medicine, Changsha City, ${ }^{3}$ Department of Oncology, Hunan \\ University of Traditional Chinese Medicine, Changsha City, Hunan Province 410000, China
}

*For correspondence: Email: wanglihuai123@163.com; Tel: +86-731-85369913

Sent for review: 3 December 2020

Revised accepted: 24 February 2021

\begin{abstract}
Purpose: To investigate the role of nuclear protein 1 (NUPR1) in the drug resistance of non-small cell lung cancer (NSCLC) and its regulatory mechanisms.

Methods: Quantitative polymerase chain reaction ( $(P C R)$ and immunoblot assays were conducted to determine NUPR1 expression in A549 cells. Cisplatin sensitivity and cisplatin-induced apoptosis were investigated in NUPR1 knockdown or overexpressed cells via 3-(4,5-dimethylthiazol-2-yl)-2,5diphenyltetrazolium bromide (MTT) assay and flow cytometry. The potential association between unfolded protein response (UPR) and NUPR1 levels in response to cisplatin were explored. The effect of endoplasmic reticulum (ER) stress on apoptosis was examined using flow cytometry.

Results: Cisplatin treatment promoted the expression of NUPR1 in NSCLC cells. NUPR1 regulated cisplatin resistance in NSCLC and also regulated UPR in ER stress induced by cisplatin. The results show NUPR1 regulated apoptosis induced by ER stress following tunicamycin treatment.

Conclusion: NSCLC cells may promote the UPR in ER stress by promoting the expression of NUPR1, thereby reducing the ER stress induced by cisplatin.
\end{abstract}

Keywords: Non-small cell lung cancer (NSCLC), Nuclear protein 1 (NUPR1), unfolded protein response (UPR), Cisplatin, Endoplasmic reticulum (ER) stress

\begin{abstract}
This is an Open Access article that uses a fund-ing model which does not charge readers or their institutions for access and distributed under the terms of the Creative Commons Attribution License (http://creativecommons.org/licenses/by/4.0) and the Budapest Open Access Initiative (http://www.budapestopenaccessinitiative.org/read), which permit unrestricted use, distribution, and reproduction in any medium, provided the original work is properly credited.
\end{abstract}

Tropical Journal of Pharmaceutical Research is indexed by Science Citation Index (SciSearch), Scopus, International Pharmaceutical Abstract, Chemical Abstracts, Embase, Index Copernicus, EBSCO, African Index Medicus, JournalSeek, Journal Citation Reports/Science Edition, Directory of Open Access Journals (DOAJ), African Journal Online, Bioline International, Open-J-Gate and Pharmacy Abstracts

\section{INTRODUCTION}

Lung cancer is a type of malignant tumor with high morbidity and mortality, making it a great threat to human health. Non-small cell lung cancer (NSCLC) accounts for about $80 \%$ of lung cancer [1]. The morbidity and mortality of NSCLC are highest in men. The main treatment methods of NSCLC include surgical resection, chemoradiotherapy, and targeted therapy [2]. Surgical resection is often unable to eradicate the cancer, leading to tumor recurrence, but the 
drug resistance and side effects of chemoradiotherapy still need to be resolved [3]. Much progress has been made in understanding targeted therapy as an effective treatment for NSCLC, but further investigation is still required.

The endoplasmic reticulum (ER) is an important organelle in eukaryotic cells [4] where protein are synthesized, folded, and secreted [5]. The ER has a number of cellular functions [6]. ER stress can trigger unfolded protein response (UPR) [7]. In tumor cells, adaptive UPR correct ER stress and maintain tumor growth and proliferation by processing unfolded or misfolded proteins.

The unfolded protein response (UPR) is one of the main mechanisms of tumor cell tolerance to chemotherapeutic drugs [8]. Rapid proliferation leads to an environment consisting of ischemia, hypoxia, and malnutrition in tumors. A large number of unfolded or misfolded proteins accumulate in the ER to activate the UPR process [9]. Under mild ER stress, UPR could further promote the proliferation of a tumor and increase its tolerance to chemotherapy [10].

Nuclear protein 1 (NUPR1) functions as a transcriptional regulator, and affects multiple cellular functions such as regulation of the DNA damage response, apoptosis, autophagy, and cell cycle in response to various types of cellular stressors [11]. The role of NUPR1 in cancer progression and metastasis has also been widely studied [12]. NUPR1 also plays an important role in tumor resistance.

The aim of this study was to investigate the involvement of NUPR1 in the drug resistance of NSCLC and its regulatory mechanisms.

\section{EXPERIMENTAL}

\section{Cell culture}

A549 cells obtained from ATCC (Gaithersburg, MD, USA) were maintained in Dulbecco's modified essential medium (DMEM) supplemented with $10 \%$ fetal bovine serum (FBS, Gibco, CA, USA).

For NURP1 knockdown, NUPR1 shRNA \#1 and \#2 were transfected into A549 cells with Lipofectamine 3000 (Invitrogen, Carlsbad, CA, USA). For NURP1 overexpression, the NUPR1 gene was amplified using the primers shown in Table 1 and ligated into the pcDNA3.1 plasmid. Cisplatin and the ER stress agent tunicamycin were purchased from Sigma-Aldrich (SigmaAldrich, St Louis, MO, USA).

\section{Quantitative PCR assays}

Trizol (15596026, Invitrogen) reagent was used for total RNA isolation from tissues and cells. MMLV reverse transcriptase (M1701, Promega, Wisconsin, USA) was utilized to transcribe total RNA into cDNA. Quantitative PCR was performed using the SYBR Ex Taq kit (638319, Takara, Japan), and GAPDH was used as internal control. The trimers used are listed in Table 2.

\section{Immunoblot assays}

Proteins were extracted with RIPA lysis buffer and separated using SDS-PAGE. The proteins were transferred onto PVDF membrane, and then the membranes were immersed in 5\% BSA in TBST and subsequently incubated using specific antibodies against NUPR1 (1:500 dilution, Santa Cruz Biotechnology, Inc. Dallas, TX, USA), CLAR (1:500 dilution, Santa Cruz Biotechnology), GADD153/CHOP (1:1000 dilution, Santa Cruz Biotechnology), PERK (1:500 dilution, Santa Cruz Biotechnology), $\beta$ actin (1:2000 dilution, Santa Cruz Biotechnology), IRE1 (Cell Signaling, Danvers, MA, USA), and GRP78 (1:1000 dilution, Abcam, Cambridge, UK).

Then, the membranes were incubated with HRPconjugated secondary antibodies in TBST buffer for $1 \mathrm{~h}$ before incubation with chemiluminescence reagents.

Table 1: Primer sequences used in this study

\begin{tabular}{ll}
\hline Variable & Sequence \\
\hline NUPR1 shRNA \#1 & 5'-CCGGGGATGAATCTGACCTCTATACCTC-3', 5'- \\
plasmid & GAGCTATAGAGGTCAGATTCATCCTTTTTG-3' \\
NUPR1 shRNA \#2 & 5'-CCGGTGGACACTACACCCAGCAATACTC-3', 5'- \\
plasmid & GAGTATTGCTGGGTGTAGTGTCCATTTTTG-3' \\
NUPR1 & 5'-TGGATCCACCATGGCCACCTTCCCA -3' \\
overexpression & 5'-TCTCGAGGCGCCGTGCCCCT-3' \\
\hline
\end{tabular}


Table 2: Trimer sequences used in this study

\begin{tabular}{ll}
\hline Variable & Sequence \\
\hline NURP1-forward & 5'-CTGGATGAATCTGACCTCTA-3' \\
NURP1-reverse & 5'-CGCTTCTTCCTCTCTGAATT-3' \\
CHOP-forward & 5'-GTCCAGCTGGGAGCTGGAAG-3' \\
CHOP-reverse & 5'-CTGACTGGAATCTGGAGAG-3', \\
XBP1-forward & 5'-ACACGCTTGGGAATGGACAC-3', \\
XBP1-reverse & 5'-CCATGGGAAGATGTTCTGGG3', \\
GAP78-forward & 5'-GCTCGACTCGAATTCCAAAG-3' \\
GAP78-reverse & 5'-TTTGTCAGGGGTCTTTCACC-3' \\
PERK-forward & 5'-ATCCCCCATGGAACGACCTG-3-3' \\
PERK-reverse & 5'-ACCCGCCAGGGACAAAAATG-3' \\
CLAR-forward & 5'-GATCAAGCTTGTCTGGATAAATCACAC-3' \\
CLAR-reverse & 5'-GATCGTCGACCACTCCACCTCAAAACAAG-3' \\
beta-actin-forward & 5'-CCTTCCTGGGCATGGAGTCCTGT-3' \\
beta-actin-reverse & 5'-GGAGCAATGATCTTGATCTTC-3' \\
\hline
\end{tabular}

\section{MTT assays}

Cells were seeded into 96-well plates at about $10^{3}$ cells per well. After culturing for $24 \mathrm{~h}$, MTT solution was incubated in each well for $6 \mathrm{~h}$ and then removed. Then dimethyl sulfoxide (DMSO) was used to extract the dissolved cells. The OD value was measured at $562 \mathrm{~nm}$ wavelength using a microplate reader.

\section{Flow cytometry}

Cells were washed with PBS buffer. After centrifugation at $1000 \mathrm{~g}$, cells were resuspended in binding buffer with Annexin V-FITC and propidium iodide $(\mathrm{PI})$ and then incubated for 10 min. The cells were subjected to flow cytometric analysis to determine the percentage of apoptotic cells using a FACS Calibur (BD Biosciences, San Jose, CA, USA) flow cytometer.

\section{Statistics}

Data are presented as mean \pm standard deviation (SD). Statistical analyses were conducted using the Student's $t$-test. A $p$-value < 0.05 was considered statistically significant difference.

\section{RESULTS}

\section{Cisplatin treatment promotes the expression of NUPR1 in NSCLC cells}

To investigate the role of NUPR1 in the drug resistance of NSCLC, the expression of NUPR1 mRNA was assessed in cisplatin-treated A549 cells. Nupr1 mRNA was enhanced in cisplatininduced cells after cisplatin addition for $24 \mathrm{~h}$. Cisplatin promoted NUPR1 expression in a timedependent manner (Figure $1 \mathrm{~A}$ and $\mathrm{B}$ ). Maximum expression was observed with $30 \mu \mathrm{g} / \mathrm{ml}$ cisplatin treatment. These results demonstrate that cisplatin treatment promotes the expression of NUPR1 in NSCLC cells.
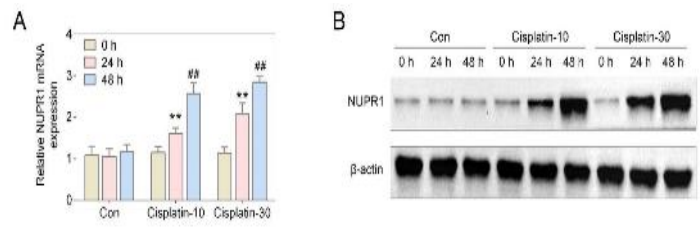

Figure 1: Cisplatin treatment promotes the expression of NUPR1 in NSCLC cells. (A) qPCR was performed to assess the level of NUPR1 mRNA in cells stimulated with 10 or $30 \mu \mathrm{g} / \mathrm{ml}$ cisplatin for 24 and 48 h. (B) Immunoblot was conducted to examine the level of NUPR1 protein in cells stimulated with 10 or 30 $\mu \mathrm{g} / \mathrm{ml}$ cisplatin for 24 and $48 \mathrm{~h} ;{ }^{* \star} p<0.01$ vs control; \#\# $p 0.01$ vs cisplatin at $24 \mathrm{~h}$

\section{NUPR1 regulates cisplatin resistance in NSCLC cells}

Since NUPR1 was upregulated in cisplatintreated cells, we proceeded to investigate the function of NUPR1 in cisplatin resistance. To this end, we examined the function of NUPR1 in cisplatin sensitivity and apoptosis through NUPR1 depletion or overexpression in A549 cells. As shown in Figure 2 A, NUPR1 was depleted or enhanced following transfection with NUPR1 shRNA or NUPR1 overexpressing plasmid, respectively. NUPR1 knockdown significantly increased cisplatin sensitivity with a lower $\mathrm{IC}_{50}(3.689 \mu \mathrm{g} / \mathrm{ml}$ in NUPR1 knockdown cells versus $8.418 \mu \mathrm{g} / \mathrm{mL}$ in control cells), while NUPR1 overexpression led to defective cisplatin sensitivity with a higher $\mathrm{IC}_{50}$ of cisplatin (14.66 $\mu \mathrm{g} / \mathrm{ml}$ in NUPR1 overexpressed cells versus $8.411 \mu \mathrm{g} / \mathrm{ml}$ in control cells) (Figure $2 \mathrm{~B}$ ). UPR1 knockdown was also found to induce apoptosis upon treatment with $5 \mu \mathrm{g} / \mathrm{ml}$ cisplatin according to a previous $I_{50}$ value. On the contrary, NUPR1 overexpression reduced the apoptotic cell ratio (Figure $2 \mathrm{C}$ ). Taken together, our data suggest 
that NUPR1 affects cisplatin sensitivity in NSCLC cells.
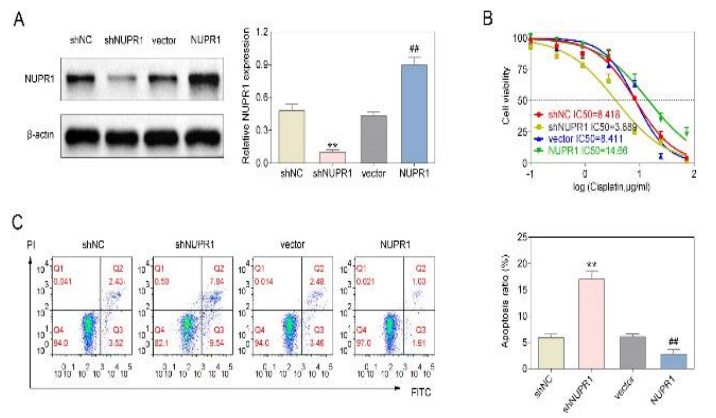

Figure 2: NUPR1 regulates cisplatin resistance in NSCLC cells. (A) The efficiency of NUPR1 knockdown and overexpression were validated by immunoblot analysis. (B) Cell viability following NUPR1 knockdown or overexpression was evaluated by MTT assay. (C) The apoptotic ratio in NUPR1 level manipulation group was determined by flow cytometry; ${ }^{* *} p<0.01$; ${ }^{\# \#} p<$ 0.01

\section{NUPR1 regulates UPR in ER stress induced by cisplatin}

The UPR is one of the main mechanisms of tumor cell tolerance to chemotherapeutic drugs [13]. Thus, we hypothesized that NUPR1 mediates cisplatin resistance via UPR in ER stress. As shown in Figures $3 \mathrm{~A}$ and $\mathrm{B}$, after the addition of $5 \mu \mathrm{g} / \mathrm{mL}$ cisplatin for $24 \mathrm{~h}$, NUPR1 knockdown significantly downregulated both mRNA and protein levels of XBP1, CLAR, PERK, IREa, and CHOP. The level of glucose regulated protein 78 (GRP78) was minimally affected by NUPR1 shRNA transfection. Conversely, NUPR1 overexpression elevated the mRNA and protein levels of XBP1, CLAR, PERK, IREa, CHOP, and GRP78 (Figure $3 \mathrm{~A}$ and $\mathrm{B}$ ). These results demonstrate that NUPR 1 modulates the UPR in ER stress induced by cisplatin.

\section{NUPR1 regulated apoptosis resulting from tunicamycin-induced ER stress}

To investigate whether ER stress-induced apoptosis was regulated by NUPR1, A549 cells were treated with the ER stress-inducing agent tunicamycin at $1 \mu \mathrm{M}$ for $24 \mathrm{~h}$. NUPR1 knockdown reduced XBP1, PERK, IRE $\alpha$, CHOP, and GRP78 protein level in A549 cells with tunicamycin treatment. NUPR1 overexpression increased CLAR, IRE $\alpha$, CHOP, and GRP78 protein level (Figure 4A). Flow cytometry was performed to analyze the apoptotic ratio in NUPR1-depleted or -overexpressed A549 cells. NUPR1 depletion led to a higher apoptotic ratio in A549 cells, while NUPR1 overexpression inhibited apoptosis in A549 cells treated with tunicamycin (Figure 4B).
Collectively, our data demonstrate that NUPR1 may be a regulator in tunicamycin-induced apoptosis.
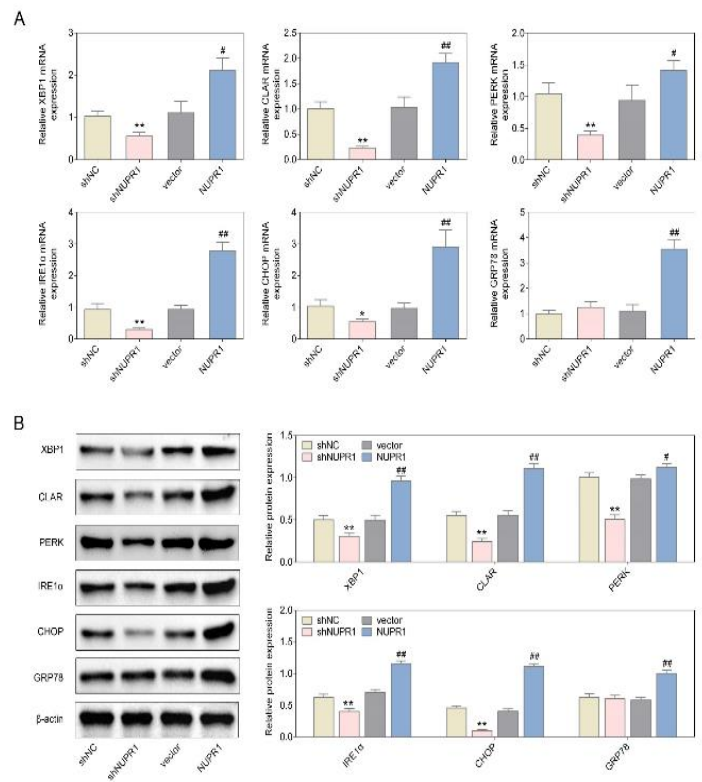

Figure 3: NUPR1 regulates the UPR in ER stress induced by cisplatin. (A) The mRNA level of ER stressrelated proteins was measured in NUPR1-depleted or ectopically expressed cells stimulated with cisplatin by qPCR. (B) The level of ER stress-related proteins was assessed in NUPR1 depleted or ectopically expressed cells stimulated with cisplatin by immunoblot analysis; ${ }^{* *} p<0.01 ;{ }^{\# \#} p<0.01$

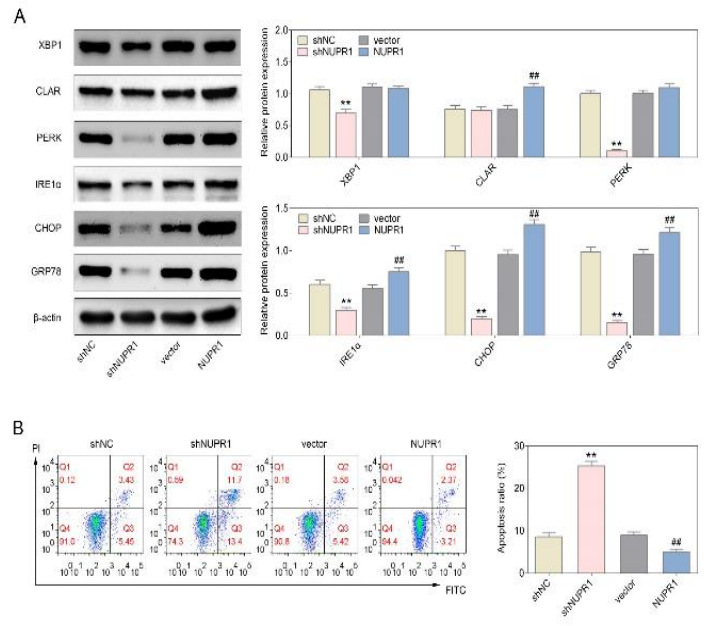

Figure 4: NUPR1 regulates apoptosis resulting from tunicamycin-induced ER stress. (A) The level of ER stress-related proteins was assessed in NUPR1depleted or ectopically expressed cells treated with tunicamycin. (B) The apoptotic ratio in NUPR1depleted or ectopically expressed cells treated with tunicamycin were determined using flow cytometry; ${ }^{* *} p<0.01 ;{ }^{\# \#} p<0.01$

Trop J Pharm Res, March 2021; 20(3): 522 


\section{DISCUSSION}

There are about 500,000 new cases of lung cancer in the world every year, $80 \%$ of which are SCLC [14]. Early diagnosis of NSCLC is difficult in clinics. About $50 \%$ of NSCLC patients have distant metastasis. Cisplatin is an effective and widely used first-line drug [3]. Elucidating the molecular mechanism of chemotherapy resistance in NSCLC is important to improve the prognosis of NSCLC patients. NUPR1 protein has been shown to regulate cisplatin resistance in NSCLC cells. Therefore, NUPR1 could serve as a promising therapeutic target for cisplatinresistance NSCLC.

NUPR1 protein regulates multiple types of signaling pathways and therefore causes varying effects on cancer progression and metastasis. Its role in some cancers has been revealed; however, the role of NUPR1 remains poorly understood in other types of cancers. NUPR 1 mediates oncogenic potential and chemotherapeutic resistance in cancer. The data in this study confirmed the role of NUPR1 in chemotherapeutic resistance. Previous studies further confirmed that NUPR1 could affect chemotherapeutic resistance by regulating antiapoptotic activity and autophagy. This study confirmed NUPR1 regulates apoptosis caused by tunicamycin-induced ER stress, and might therefore affect the chemotherapeutic resistance of NSCLC. Previous studies investigated the role of NUPR1 in breast, lung, and colorectal cancer progression. Administration of the inhibitor of NUPR1, ZZW-115, is known as an efficient strategy for the treatment of hepatocellular carcinoma (HCC). These studies, together with our findings, suggest the potential of NUPR1 as a cancer therapeutic target.

The UPR often leads to ER stress, which affects tumor progression [13]. In cells, the ER is responsible for the proper folding and processing of polypeptide chains into functional proteins. Some exogenous or endogenous factors lead to UPR, which will have a direct impact on tumor proliferation, migration, and apoptosis, and the associations between UPR and ER stress, autophagy, and apoptosis have already been revealed [8]. Several proteins affect the apoptosis and autophagy of cancer cells via UPR-induced ER stress. For example, ATMassociated signaling triggers the UPR and cell death in response to ER stress [15]. Misfolded proteins could bind and activate DR5 to trigger apoptosis during unresolved ER stress [16]. Another study showed that autophagosome accumulation-mediated ATP energy deprivation triggered nonapoptotic cell death in lung cancer by promoting UPR [17]. However, the precise regulatory mechanism needs further study.

In clinical practice, cisplatin-based chemotherapy remains one of the main treatments for NSCLC even though inherent or acquired drug resistance limits the clinical application of cisplatin [18]. In recent years, good results have been achieved in NSCLC in vitro [3]. This study revealed that targeting NUPR1 could be an effective treatment for cisplatin resistance in NSCLC. Investigation into the possibility of NUPR1 as a therapeutic target is still needed to better understand cisplatin resistance in NSCLC at the animal level.

\section{CONCLUSION}

The findings of this study indicate that NUPR1 knockdown increases sensitivity to cisplatin, while NUPR1 overexpression improves tolerance towards cisplatin. NUPR1 promotes UPR in ER stress by enhancing the expression of NUPR1, thereby reducing ER stress induced by cisplatin. Thus, NUPR1 might serve as a potential therapeutic target for NSCLC drug resistance.

\section{DECLARATIONS}

\section{Acknowledgement}

This work was supported by the Outstanding Youth Project of Hunan Provincial Department of Education (Grant no. 19B419) and Hunan Provincial Health Commission Project (Grant no. C2019092).

\section{Competing interests}

There are no conflicts of interest to disclose.

\section{Contribution of authors}

We declare that this work was performed by the authors named in this article and all liabilities pertaining to claims relating to the content of this article will be borne by the authors. Yinhui Sun and Lihuai Wang designed the study, as well as supervised the data collection. Changqing Deng and Hua Liu analyzed and interpreted the data. Zhongcong Guo and Zelin Chang prepared the manuscript for publication and reviewed the draft. All authors have read and approved the manuscript.

\section{Open Access}

This is an Open Access article that uses a funding model which does not charge readers or their institutions for access and distributed under 
the terms of the Creative Commons Attribution License (http://creativecommons.org/licenses/by/ 4.0) and the Budapest Open Access Initiative (http://www.budapestopenaccessinitiative.org/rea d), which permit unrestricted use, distribution, and reproduction in any medium, provided the original work is properly credited.

\section{REFERENCES}

1. Lan $H$, Hao $Y, L v Y, L i G$, Mo Y, Zheng C, Li Y. Synergistic effect of a combination of granulocyte macrophage colony-stimulating factor and thymosin a1 on Lewis lung cancer transplanted tumor in mice. Trop J Pharm Res 2020; 19: 759-764.

2. LiU B, Chen W, Li H, Li F, Jin X, Li Q. Radiosensitization of NSCLC cells to $X$-rays and carbon ions by the CHK1/CHK2 inhibitor AZD7762, Honokiol and Tunicamycin. Radiat Environ Biophys 2020.

3. Lin Z, Pan J, Chen L, Wang X, Chen Y. MiR-140 Resensitizes Cisplatin-Resistant NSCLC Cells to Cisplatin Treatment Through the SIRT1/ROS/JNK Pathway. Onco Targets Ther 2020; 13: 8149-8160.

4. Martinez-Klimova E, Aparicio-Trejo OE, Gomez-Sierra $T$, Jimenez-Uribe AP, Bellido B, Pedraza-Chaverri J. Mitochondrial dysfunction and endoplasmic reticulum stress in the promotion of fibrosis in obstructive nephropathy induced by unilateral ureteral obstruction. Biofactors 2020.

5. Brown M, Dainty S, Strudwick N, Mihai AD, Watson JN, Dendooven $R$, Paton AW, Paton JC, Schroder M. Endoplasmic reticulum stress causes insulin resistance by inhibiting delivery of newly synthesised insulin receptors to the cell surface. Mol Biol Cell 2020: mbcE18010013.

6. de Seny D, Bianchi E, Baiwir D, Cobraiville G, Collin C, Deliege M, Kaiser MJ, Mazzucchelli G, Hauzeur JP, Delvenne $P$ et al. Proteins involved in the endoplasmic reticulum stress are modulated in synovitis of osteoarthritis, chronic pyrophosphate arthropathy and rheumatoid arthritis, and correlate with the histological inflammatory score. Sci Rep 2020; 10(1): 14159.

7. Song $Q$, He Z, Li B, LiU J, LiU L, Liao W, Xiong Y, Song $C$, Yang S, Liu Y. Melatonin inhibits oxalate-induced endoplasmic reticulum stress and apoptosis in HK-2 cells by activating the AMPK pathway. Cell Cycle 2020: 1-11.

8. Oh JY, Lee YJ, Sai S, Ohno T, Kong CB, Lim SH, Kim EH. The Unfolded Protein Response: Neutron-Induced
Therapy Autophagy as a Promising Treatment Option for Osteosarcoma. Int J Mol Sci 2020; 21(11).

9. Wang S, Ma X, Wang H, He H. Induction of the Unfolded Protein Response during Bovine Alphaherpesvirus 1 Infection. Viruses 2020; 12(9).

10. Yu L, Wang L, Kim JE, Mao C, Shapiro DJ. Src couples estrogen receptor to the anticipatory unfolded protein response and regulates cancer cell fate under stress. Biochim Biophys Acta Mol Cell Res 2020; 1867(10): 118765.

11. Pommier RM, Gout J, Vincent DF, Cano CE, Kaniewski B, Martel S, Rodriguez J, Fourel G, Valcourt U, Marie $J C$ et al. The human NUPR1/P8 gene is transcriptionally activated by transforming growth factor beta via the SMAD signalling pathway. Biochem J 2012; 445(2): 285-293.

12. Li J, Ren S, Liu Y, Lian Z, Dong B, Yao Y, Xu Y. Knockdown of NUPR1 inhibits the proliferation of glioblastoma cells via ERK1/2, p38 MAPK and caspase3. J Neurooncol 2017; 132(1): 15-26.

13. Ghemrawi R, Khair M. Endoplasmic Reticulum Stress and Unfolded Protein Response in Neurodegenerative Diseases. Int J Mol Sci 2020; 21(17).

14. Ernani V, Appiah AK, Baine MJ, Smith LM, Ganti AK. The impact of histology in the outcomes of patients with early-stage non-small cell lung cancer (NSCLC) treated with stereotactic body radiation therapy (SBRT) and adjuvant chemotherapy. Cancer Treat Res Commun 2020; 24: 100197.

15. Hotokezaka Y, Katayama I, Nakamura T. ATMassociated signalling triggers the unfolded protein response and cell death in response to stress. Commun Biol 2020; 3(1): 378.

16. Pistritto G, Puca R, Nardinocchi L, Sacchi A, D'Orazi G. HIPK2-induced p53Ser46 phosphorylation activates the KILLER/DR5-mediated caspase-8 extrinsic apoptotic pathway. Cell Death Differ 2007; 14(10): 1837-1839.

17. Chou CW, Yang RY, Chan LC, Li CF, Sun L, Lee HH, Lee $P C$, Sher YP, Ying $H$, Hung MC. The stabilization of $P D-L 1$ by the endoplasmic reticulum stress protein GRP78 in triple-negative breast cancer. Am J Cancer Res 2020; 10(8): 2621-2634.

18. Papadimitrakopoulou VA, Mok TS, Han JY, Ahn MJ, Delmonte A, Ramalingam SS, Kim SW, Shepherd FA, Laskin J, He $Y$ et al. Osimertinib versus platinumpemetrexed for patients with EGFR T790M advanced NSCLC and progression on a prior EGFR-tyrosine kinase inhibitor: AURA3 overall survival analysis. Ann Oncol 2020; 31(11): 1536-1544. 